\title{
Seleção simultânea em progênies de sorgo-sacarino por meio de índices de seleção
}

\author{
Álvaro Eugênio Duarte de França( ${ }^{(1)}$, Rafael Augusto da Costa Parrella(2), Vander Fillipe de Souza(2), \\ Gerson Quirino Bastos ${ }^{(1)}$, José Airton Rodrigues Nunes ${ }^{(3)}$ e Robert Eugene Schaffert ${ }^{(2)}$
}

(1)Universidade Federal Rural de Pernambuco, Departamento de Agronomia, Rua Manuel de Medeiros, s/no, Dois Irmãos, CEP 52171-900 Recife, PE, Brasil. E-mail: alvarofranca@hotmail.com, bastosgq@hotmail.com (2)Embrapa Milho e Sorgo, Rodovia MG-424, Km 45, Zona Rural, CEP 35701-970 Sete Lagoas, MG, Brasil. E-mail: rafael.parrella@embrapa.br, vanderfsouza@gmail.com, robert.schaffert@embrapa.br ${ }^{(3)}$ Universidade Federal de Lavras, Departamento de Biologia, Campus Universitário, Caixa Postal 3037, CEP 37200-000 Lavras, MG, Brasil. E-mail: jarnunes@dbi.ufla.br

Resumo - O objetivo deste trabalho foi comparar ganhos genéticos obtidos por diferentes índices de seleção aplicados a múltiplos caracteres agronômicos de sorgo-sacarino (Sorghum bicolor). Foram feitas avaliações de 189 progênies F5 e sete testemunhas, em delineamento de blocos incompletos, com três repetições. Mensuraram-se os caracteres produtividade de matéria verde, teor de sólidos solúveis totais, extração de caldo, teor de sacarose no caldo e sólidos solúveis totais por hectare. Analisaram-se os índices de soma de postos de Mulamba e Mock (ou índice de ranks), da soma dos valores padronizados (ou índice Z), índice padronizado pela média e índice padronizado pela média modificado. As correlações e as percentagens de coincidência na seleção das progênies foram elevadas entre o índice $\mathrm{Z}$, o índice padronizado pela média e o índice padronizado pela média modificado. Entre os índices de seleção testados, o de soma de postos de Mulamba e Mock foi o menos eficiente. A seleção simultânea de progênies de sorgo-sacarino permite ganhos genéticos expressivos para todos os caracteres mensurados.

Termos para indexação: Sorghum bicolor, ganho genético, melhoramento genético de plantas.

\section{Simultaneous selection in sweet sorghum progenies by selection indices}

Abstract - The objective of this work was to compare the genetic gains obtained by different selection indices applied to various agronomic traits of sweet sorghum (Sorghum bicolor). Evaluations were carried out for 189 F5 progenies and seven controls, in an incomplete block design, with three replicates. The measured traits were green matter yield, content of total soluble solids, juice extraction, sucrose content in juice, and total soluble solids per hectare. The rank summation index of Mulamba and Mock (or index of ranks), standardized value summation index (or $\mathrm{Z}$ index), standardized by the mean index, and the modified standardized by the mean index were analyzed. The correlations and coincidence percentages in the progeny selections were high between the $\mathrm{Z}$ index, the standardized by the mean index, and the modified standardized by the mean index. Among the tested selection indices, the rank summation index of Mulamba and Mock was the less efficient. The simultaneous selection of sweet sorghum progenies allows expressive genetic gains for all measured traits.

Index terms: Sorghum bicolor, genetic gain, plant breeding.

\section{Introdução}

O sorgo-sacarino, Sorghum bicolor (L.) Moench, é uma cultura bioenergética que apresenta colmos ricos em açúcares fermentescíveis e com potencial para a produção de etanol na mesma instalação utilizada pela cana-de-açúcar (Anandan et al., 2012). Além disso, o sorgo-sacarino apresenta como vantagens o ciclo curto de produção, propagação por sementes, facilidade de mecanização, menor exigência de insumos e tolerância à seca (Durães, 2011).
Para otimizar o melhoramento do sorgo-sacarino para a produção de etanol, as características agronômicas e tecnológicas precisam ser melhoradas simultaneamente. Assim, a utilização de índices de seleção permite combinar as múltiplas informações dos caracteres e contribui para a seleção dos genótipos superiores com base em um conjunto complexo de variáveis (Rodrigues et al., 2011).

$\mathrm{Na}$ escolha do índice de seleção, deve-se considerar a facilidade de interpretação. O índice da soma de postos ou "ranks", proposto por Mulamba \& Mock (1978), por 
ser um índice não paramétrico, apresenta a vantagem de não necessitar de pesos econômicos nem da estimação de parâmetros além das médias. $\mathrm{O}$ índice da soma de postos é baseado no ordenamento dos genótipos quanto ao caráter desejado e, posteriormente, na soma destes postos baseada nos múltiplos caracteres (Teixeira et al., 2012). $O$ índice $Z$ permite padronizar os dados a serem utilizados como uma alternativa à seleção e não necessita de estimativas de covariâncias genéticas e fenotípicas (Ramalho et al., 2012). Entretanto, o índice $Z$ é composto pela soma das variáveis $Z$, e estas podem assumir tanto um valor negativo quanto um positivo, o que dificultaria a análise do melhorista (Reis et al., 2011). Uma alternativa para se obter um índice composto pelas variáveis de interesse, que apresente valores exclusivamente positivos, seria a padronização das variáveis pela média. Além disso, de acordo com a priorização dos caracteres, podem-se atribuir pesos econômicos para cada variável.

Os índices são utilizados para a seleção de genótipos baseados em múltiplos caracteres, contudo, há a possibilidade de ganho genético negativo de um caráter individual e de seleção de genótipos não desejáveis para determinado caráter (Krause et al., 2012). Um método condicionado à eliminação de valores negativos pode ser empregado, a fim de que haja apenas ganhos genéticos positivos em cada caráter separadamente, em cada progênie selecionada. Assim, a comparação dos índices de seleção possibilita a seleção de genótipos superiores com base em múltiplos caracteres, o que ainda é necessário ser feito para a cultura do sorgo-sacarino.

O objetivo deste trabalho foi comparar ganhos genéticos, obtidos por diferentes índices de seleção aplicados a múltiplos caracteres agronômicos de sorgo-sacarino.

\section{Material e Métodos}

O experimento foi realizado na área experimental da Embrapa Milho e Sorgo, no Município de Sete Lagoas, MG, a $19^{\circ} 27^{\prime} 57^{\prime \prime}$ S, $44^{\circ} 14^{\prime} 49^{\prime \prime} \mathrm{W}$ e a $767 \mathrm{~m}$ de altitude. A região apresenta clima tropical chuvoso, do tipo Aw, de acordo com a classificação climática de Köppen-Geiger, com média anual em torno de $23^{\circ} \mathrm{C}$ e média máxima de $28^{\circ} \mathrm{C}$. O período chuvoso vai de outubro a março, com índice pluvial médio anual de $1.403 \mathrm{~mm}$.

Os tratamentos avaliados consistiram de 189 progênies $\mathrm{F}_{5}$, provenientes de 16 subpopulações resultantes de 16 cruzamentos biparentais, e de sete testemunhas, das quais dois híbridos comerciais CV198 e CV568, duas cultivares BRS508 e BRS511, e três materiais promissores CMSXS643, CMSXS646 e CMSXS647, no total de 196 tratamentos. Os tratamentos foram alocados nas parcelas, em delineamento experimental de blocos incompletos, com três repetições. As parcelas constituíram-se de duas linhas de $3 \mathrm{~m}$, espaçadas $0,3 \mathrm{~m}$ entre as linhas e $0,5 \mathrm{~m}$ entre as parcelas, em que uma linha foi colhida para avaliação dos caracteres agronômicos, e a outra para as análises laboratoriais. A densidade de plantio utilizada foi de 10 plantas por metro linear após o desbaste.

A semeadura foi realizada em 14 de fevereiro de 2014, com irrigação por pivô central e lâmina de irrigação de $10 \mathrm{~mm}$ semanais, de modo a evitar o estresse hídrico da cultura. A colheita foi realizada de 15 a 17 de junho de 2014, quando todas as progênies apresentaram grau de maturidade desejável.

Mensuraram-se os seguintes caracteres agronômicos: produtividade de massa de matéria verde por hectare (PMV), obtida a partir do corte e pesagem das plantas, sem as panículas, da área útil da parcela a 5,0 cm da superfície do solo estimada para hectare; teor de sólidos solúveis totais (BRIX), determinado por meio de refratômetro de laboratório, com correção de temperatura automática, em graus Brix (Consecana, 2006); extração de caldo (EXT), determinado pela relação entre a massa de caldo extraído com uso do moedor de cana modelo B721 Rolos Inox, com motor à gasolina, e a massa de colmos; teor de sacarose aparente no caldo, obtido com uso do polarímetro digital de peso normal (POL) (Consecana, 2006); e toneladas de sólidos solúveis totais, em graus Brix, por hectare (TBH), que foi obtido pelo produto entre PMV, BRIX e EXT (Pedrozo et al., 2008; Lombardi et al., 2015).

Os dados foram analisados com a abordagem de modelos mistos REML/BLUP (máxima verossimilhança restrita e melhor predição linear não viesada), de acordo com o modelo estatístico $\mathrm{y}=\mathrm{Xr}+\mathrm{Zg}+\mathrm{Wb}+\varepsilon$, em que: y é o vetor de dados; $\mathrm{r}$ é o vetor dos efeitos de repetição, considerados como fixos, somados à média geral; $g$ é o vetor dos efeitos genotípicos, considerados como aleatórios; $b$ é o vetor dos efeitos de blocos, considerados como aleatórios; e $\varepsilon$ é o vetor de erros ou resíduos (aleatórios). As letras maiúsculas representam as matrizes de incidência para os referidos efeitos (Resende, 2007). 
Estimaram-se os seguintes parâmetros genéticos: Vg, variância genotípica; Ve, variância residual; Vf, variância fenotípica; $h^{2}$, herdabilidade ajustada da média do genótipo; acurácia; $\mathrm{CVg} \%$, coeficiente de variação genotípica; $\mathrm{CVe} \%$, coeficiente de variação residual; e $\mathrm{CVr}$, coeficiente de variação relativa, obtido pela relação $\mathrm{CVg} \%$ / $\mathrm{CVe} \%$. As correlações genéticas entre os caracteres foram estimadas a partir das predições BLUP de cada progênie (Resende, 2007).

A seleção simultânea dos caracteres foi feita com base no índice da soma de postos ou índice de ranks (IR), índice da soma das variáveis padronizadas ou índice Z (IZ), índice padronizado pela média (IPM) e índice padronizado pela média modificado (IPMM). Além dos índices, testou-se um modo de seleção combinada em vários caracteres - denominado de seleção de ganhos positivos (SGP) -, de modo que cada progênie selecionada fosse maior do que a média de cada caráter utilizado na seleção (Mendes et al., 2009; Cruz et al., 2012; Ramalho et al., 2012).

O índice de soma de postos ou índice de ranks (IR) é realizado com base no ranqueamento de cada tratamento para múltiplos caracteres. Considera-se o modelo do índice $\mathrm{Ij}=\Sigma$ nij, em que: Ij é o índice para o genótipo j; e nij é o número de classificação do caráter i, para o genótipo j. O índice $\mathrm{Z}$ é implementado a partir da padronização dos dados das parcelas ou dos tratamentos e permite a visualização do desempenho de cada progênie para todos os caracteres (Mendes, 2009). Este índice tem como fundamento a padronização das variáveis (Zij), com o objetivo de torná-las diretamente comparáveis pelo estimador Zijk $=(y i j-y \times j k) / s j k$, em que: Zijk é o valor da variável $\mathrm{k}$ padronizada, correspondente ao caráter $\mathrm{k}$ da população i, na repetição j; yijk é a observação do caráter $\mathrm{k}$ da população i, na repetição j; $\mathrm{y} \times$ jk é a média geral do caráter $\mathrm{k}$, na repetição j; sjk é o desvio-padrão fenotípico do caráter $k$, na repetição j. Para obter o valor do índice para todos os caracteres utilizados, basta considerar sua somatória do valor de $Z$ em cada caráter: $\Sigma Z i j k=Z i j 1+Z i j 2+\ldots+$ Zijn.

O índice padronizado pela média (IPM) é um índice de fácil obtenção, que consiste da padronização de cada tratamento para um dado caráter, pela média do experimento. $\mathrm{O}$ somatório desses valores pode ser considerado como um índice de seleção para caracteres múltiplos e é calculada pelo seguinte modelo: IPM(i) = $\Sigma($ yki $/$ ymk $)=(y 1 i / y m 1)+(y 2 i / y m 2)+\ldots+(y n i / y m n)$, em que: IPM(i) é o valor do índice associado ao genótipo i; yki é a média do indivíduo i relativo ao caracter $\mathrm{k}$; ymk é a média aritmética de todos os indivíduos/progênies em relação ao caráter $\mathrm{k}$.

A fim de destacar a importância de algum caráter sobre os demais ou determinar o peso de cada caráter, de acordo com o desejo do melhorista, o índice pode ser obtido pelo produto com um peso pré-determinado. A fórmula do índice modificado seria: $\operatorname{IPMM}(\mathrm{i})=$ $\Sigma \mathrm{ux}(\mathrm{yki} / \mathrm{ymk})=\mathrm{u} 1(\mathrm{yli} / \mathrm{ym} 1)+\mathrm{u} 2(\mathrm{y} 2 \mathrm{i} / \mathrm{ym} 2)+\ldots+$ un(yni/ymn), em que: IPMM(i) é o valor do índice associado ao genótipo i; uk é o peso econômico do caráter k; yki é a média do indivíduo i relativo ao caráter $\mathrm{k}$; ymk é a média aritmética de todos os indivíduos/ progênies em relação ao caráter $\mathrm{k}$. Na proposta inicial da utilização de índices com pesos econômicos, estes deveriam ser obtidos em razão do valor de mercado de cada caráter, por unidade avaliada (Williams, 1962). Entretanto, há uma grande variação dos valores de mercado e, para alguns caracteres, é inviável encontrar um valor econômico (Teixeira et al., 2012). Os pesos econômicos utilizados no IPMM foram os seguintes: 1,25 , para os caracteres BRIX e PMV; 1,2, para os caracteres POL e TBH; e 1,1, para o caráter EXT, de acordo com o grau de importância estabelecido.

A seleção de ganhos positivos (SGP) foi testada para múltiplos caracteres e consiste em selecionar apenas materiais que tenham valores acima da média, para todos os caracteres considerados, o que acarreta ganho genético positivo para todos os caracteres, em uma seleção baseada em caracteres múltiplos. A seleção ocorre de maneira condicional aos ganhos positivos, o que suprime a utilização da intensidade de seleção pelo melhorista. A fórmula dessa seleção pode ser expressa como: $\mathrm{SGP}(\mathrm{i})=\Sigma(\mathrm{yki} / \mathrm{ymk})=(\mathrm{yli} / \mathrm{ym} 1)+(\mathrm{y} 2 \mathrm{i} / \mathrm{ym} 2)$ $+\ldots+$ (yni/ymn), sendo (yni/ymn) $=\mathrm{x}$ e $\mathrm{x} \geq 1$, em que: yki é a média do indivíduo i relativo ao caráter k; ymk é a média aritmética de todos os indivíduos, em relação ao caráter $\mathrm{k}$.

O ganho genético foi determinado de duas formas, uma com o ganho genético geral, a partir da seleção truncada das melhores progênies, e outra com o ganho genético das progênies selecionadas, em relação às testemunhas. A predição dos ganhos por seleção, em relação ao ganho genético geral, e a predição dos ganhos por seleção, em relação às testemunhas, foram obtidas por meio da expressão GS (\%) = GS/(média $\times 100$ ), em que GS representa o ganho por seleção e é obtido com a fórmula GS $=\mathrm{DS} \times \mathrm{h}^{2}$, em que DS representa o diferencial de seleção, e $\mathrm{h}^{2}$ corresponde

Pesq. agropec. bras., Brasília, v.51, n.10, p.1737-1743, out. 2016 DOI: 10.1590/S0100-204X2016001000005 
à herdabilidade da característica. Para a predição do GS, em relação ao ganho genético geral, o DS foi obtido pela diferença entre a média dos 40 genótipos selecionados por meio dos índices de seleção (Xm) e a média da população original (X0), ou seja, DS $=$ Xm - X0 (Cruz \& Carneiro, 2003). O DS para estimar o ganho genético em relação às testemunhas resultou da diferença entre a média das 40 progênies, selecionadas por meio dos índices de seleção (Xp), e a média das testemunhas (Xt), ou seja, DS $=\mathrm{Xp}-\mathrm{Xt}$.

Em ambos os casos, selecionaram-se 40 genótipos superiores, de acordo com cada índice utilizado, o que equivale a uma intensidade de seleção de aproximadamente $20 \%$.

Para verificar a correspondência na seleção, por meio dos índices, estimou-se a correlação de Spearman e a percentagem de coincidência na seleção. As magnitudes das correlações foram expressas como: fraca, quando $0<|\mathrm{r}|<0,30$; média, quando $0,30<|\mathrm{r}|<0,60$; forte, quando $0,60<|\mathrm{r}|<0,90$; e fortíssima, quando $0,90<|\mathbf{r}|<1$ (Silva et al., 2007).

\section{Resultados e Discussão}

Entre os caracteres avaliados, observou-se diferença significativa, de acordo com o teste razão de verossimilhança entre os tratamentos, para todas as características observadas, o que indica a existência de variabilidade genética e, por conseguinte, viabiliza a prática de seleção (Tabela 1).
A acurácia foi considerada muito alta para o caráter PMV $(0,912)$, alta para os caracteres BRIX, POL e TBH e apenas moderada para o EXT $(0,682)$. A acurácia, um dos aspectos mais relevantes para a qualidade de um experimento, representa a correlação entre o valor genotípico verdadeiro do tratamento genético e o valor estimado ou predito a partir das informações dos experimentos (Resende \& Duarte, 2007).

Os caracteres PMV, BRIX POL e TBH apresentaram estimativas de herdabilidade de $0,832,0,715,0,746$ e 0,755 , respectivamente, o que mostra uma relação direta com a acurácia, também evidenciada por Cargnelutti Filho \& Stork (2009).

Segundo Vencovsky (1987), a relação entre os coeficientes de variação genotípica e residual (CVr), também denominado de índice $b$, quando apresenta um valor igual ou superior a 1 , mostra uma condição favorável à seleção. Apenas os caracteres TBH $(1,015)$ e PMV $(1,284)$ apresentaram valores expressivos $(>1)$.

Nas estimativas de correlações genéticas entre os caracteres de progênies, todos os caracteres apresentaram valores positivos, o que é uma indicação de que o aumento de qualquer característica observada não influenciou a diminuição de outra característica (Tabela 2). Uma correlação genotípica fortíssima $(0,9482)$ foi observada entre os caracteres BRIX e POL, também observada $(0,96)$ por Guigou et al. (2011), o que é uma indicação de que apenas uma das características poderia ter sido utilizada como seleção indireta para a outra.

Tabela 1. Estimativas de parâmetros genéticos, ambientais e fenotípicos para as características avaliadas nos genótipos de sorgo-sacarino.

\begin{tabular}{|c|c|c|c|c|c|}
\hline Parâmetro & $\operatorname{PMV}\left(\mathrm{Mg} \mathrm{ha}^{-1}\right)$ & EXT & BRIX & POL & $\mathrm{TBH}\left(\mathrm{Mg} \mathrm{ha}^{-1}\right)$ \\
\hline $\mathrm{Vg}$ & $75,122 * *$ & $0,001^{* *}$ & $3,547 * *$ & $3,620 * *$ & $0,255^{* *}$ \\
\hline $\mathrm{Ve}$ & 45,551 & 0,004 & 4,236 & 3,690 & 0,248 \\
\hline Vf & 120,877 & 0,005 & 7,841 & 7,406 & 0,514 \\
\hline $\mathrm{h}^{2}$ & 0,832 & 0,465 & 0,715 & 0,746 & 0,755 \\
\hline Acurácia & 0,912 & 0,682 & 0,846 & 0,864 & 0,869 \\
\hline $\mathrm{CVg} \%$ & 24,819 & 10,604 & 14,598 & 2,498 & 35,097 \\
\hline $\mathrm{CVe} \%$ & 19,326 & 19,700 & 15,954 & 2,521 & 34,595 \\
\hline $\mathrm{CVr}$ & 1,284 & 0,538 & 0,915 & 0,991 & 1,015 \\
\hline Média Geral & 34,923 & 0,308 & 12,901 & 7,618 & 1,439 \\
\hline Média das testemunhas & 36,376 & 0,298 & 12,233 & 6,675 & 1,405 \\
\hline
\end{tabular}

**Significativo a 1\% de probabilidade, pelo teste de razão de verossimilhança ou qui-quadrado, com 1 grau de liberdade. Vg, variância genotípica; Ve, variância residual; Vf, variância fenotípica; $\mathrm{h}^{2}$, herdabilidade ajustada da média do genótipo; CVg\%, coeficiente de variação genotípica; CVe\%, coeficiente de variação residual; CVr, coeficiente de variação relativa; PMV, produtividade de matéria verde; EXT, extração de caldo; BRIX, sólidos solúveis totais; POL, teor de sacarose no caldo; TBH, massa de sólidos solúveis totais por hectare. 
Quando se considerou o ganho genético geral e o caráter PMV como base, o maior ganho genético (20,48\%) foi obtido com o índice IPMM. Em relação ao caráter BRIX, os maiores ganhos foram obtidos com os índices IZ, IPM e IPMM (Tabela 3). Para o caráter EXT, a seleção SGP apresentou o ganho genético de $3,07 \%$, superior aos demais índices; e, para o caráter POL, o maior ganho genético $(21,52 \%)$ foi observado com o índice Z. De acordo com Reis et al. (2011), o índice $\mathrm{Z}$ mostrou-se satisfatório na identificação das melhores progênies de eucalipto. Concomitantemente, com o índice IR obteveram-se os menores ganhos genéticos, para todos os caracteres baseados em uma seleção simultânea quanto aos caracteres PMV, BRIX, EXT, TBH e POL (Tabela 3).

Ao se considerar o ganho genético em relação às testemunhas, quanto ao caráter PMV, o maior ganho genético $(15,70 \%)$ foi obtido com o índice IPMM. Em relação ao caráter BRIX, observou-se o maior

Tabela 2. Correlações genéticas entre os caracteres agroindustriais de sorgo-sacarino.

\begin{tabular}{lccccc}
\hline Caráter & $\begin{array}{c}\text { PMV } \\
\left(\mathrm{Mg} \mathrm{ha}^{-1}\right)\end{array}$ & EXT & BRIX & POL & $\begin{array}{c}\text { TBH } \\
\left(\mathrm{Mg} \mathrm{ha}^{-1}\right)\end{array}$ \\
\hline PMV & - & 0,0926 & $0,4129 *$ & 0,3695 & $0,8044^{* *}$ \\
EXT & & - & 0,2738 & 0,2897 & $0,5006^{*}$ \\
BRIX & & & - & $0,9482 * *$ & $0,7373^{* *}$ \\
POL & & & & - & $0,6995^{* *}$ \\
TBH & & & & & - \\
\hline
\end{tabular}

* e **Significativo a 5 e $1 \%$ de probabilidade, respectivamente, pelo teste t. PMV, produtividade de matéria verde ; EXT, extração de caldo; BRIX, sólidos solúveis totais; POL, teor de sacarose no caldo; TBH, massa de sólidos solúveis totais.

Tabela 3. Ganhos genéticos das características quanto ao ganho genético geral, a partir da seleção truncada das melhores progênies, obtidas com os índices de seleção e a seleção de ganhos positivos.

\begin{tabular}{lccccc}
\hline Índices & \multicolumn{5}{c}{ Ganhos de seleção (\%) } \\
\cline { 2 - 6 } & $\begin{array}{c}\text { PMV } \\
\left(\mathrm{Mg} \mathrm{ha}^{-1}\right)\end{array}$ & BRIX & EXT & $\begin{array}{c}\text { TBH } \\
\left(\mathrm{Mg} \mathrm{ha}^{-1}\right)\end{array}$ & $\begin{array}{c}\text { POL } \\
(\%)\end{array}$ \\
\hline IR & 13,77 & 10,20 & 1,52 & 26,06 & 18,89 \\
IPMM & 20,48 & 11,95 & 1,86 & 35,54 & 20,90 \\
IPM & 20,17 & 12,12 & 1,84 & 35,41 & 21,19 \\
IZ & 18,40 & 12,21 & 2,28 & 34,60 & 21,52 \\
SGP & 15,87 & 10,95 & 3,07 & 32,53 & 19,50 \\
\hline
\end{tabular}

Índices de seleção: IR, o índice de ranks; IPMM, índice padronizado pela média modificado; IPM, índice padronizado pela média; IZ, índice Z; e, SGP, seleção de ganhos positivos. PMV, produtividade de matéria verde; BRIX, sólidos solúveis totais; EXT, extração de caldo; TBH, massa de sólidos solúveis totais; POL, teor de sacarose no caldo. ganho $(16,82 \%)$ com o índice Z. O maior ganho $(6,15 \%)$ foi observado na seleção SGP para o caráter EXT. Com o índice IPM, mostrou-se ganho genético de $32,12 \%$ para o caráter $\mathrm{TBH}$; e com o índice $\mathrm{Z}$, mostrou-se o maior ganho $(35,19)$ para o caráter POL. Novamente, o índice IR apresentou os menores ganhos de seleção para todos os caracteres baseados na seleção simultânea (Tabela 4), tendo-se mostrado insatisfatório em comparação aos outros índices, com os menores ganhos genéticos por seleção. Tal condição foi evidenciada, também, por Garcia \& Souza Júnior (1999), na seleção de cultivares de cana-de-açúcar, uma vez que os materiais devem ser recomendados para plantio, e todos os caracteres devem estar dentro dos níveis mínimos aceitáveis, diferentemente do que foi mostrado por Pereira et al. (2013), que obtiveram ganhos genéticos satisfatórios na seleção de cultivares de café arábica, com a utilização de IR.

As estimativas das correlações de Spearman (Tabela 5) mostraram associações negativas e elevadas entre o IR e os índices IPMM (-0,7145), IPM (-0,7145) e IZ (-0,7061), enquanto o índice $Z$ apresentou correlações fortíssimas - de 0,9894 e 0,9883 - com os índices IPMM e IPM, respectivamente, o que leva à escolha de apenas um desses índices para a seleção de progênies superiores.

Entre os índices IPMM, IPM e IZ, houve elevada percentagem de coincidência na seleção (Tabela 5), pois, o índice IPMM atingiu $100 \%$ com o índice IPM, mesmo valor mostrado por Freitas et al. (2013), que compararam estes a outros índices de seleção em milho-pipoca. No presente estudo, o índice $\mathrm{Z}$

Tabela 4. Ganhos genéticos das características, em comparação às testemunhas, obtidos com índices de seleção e seleção de ganhos.

\begin{tabular}{lccccc}
\hline Índices & \multicolumn{5}{c}{ Ganhos de seleção (\%) } \\
\cline { 2 - 6 } & $\begin{array}{c}\text { PMV } \\
\left(\mathrm{Mg} \mathrm{ha}^{-1}\right)\end{array}$ & BRIX & EXT & $\begin{array}{c}\text { TBH } \\
\left(\mathrm{Mg} \mathrm{ha}^{-1}\right)\end{array}$ & $\begin{array}{c}\text { POL } \\
(\%)\end{array}$ \\
\hline IR & 9,48 & 14,24 & 3,40 & 26,63 & 31,16 \\
IPMM & 15,70 & 16,40 & 3,55 & 35,97 & 34,29 \\
IPM & 15,53 & 16,67 & 3,54 & 36,12 & 34,82 \\
IZ & 14,31 & 16,82 & 4,01 & 35,85 & 35,19 \\
SGP & 11,92 & 15,49 & 4,81 & 33,75 & 32,88 \\
\hline
\end{tabular}

Índices de seleção: IR, índice de ranks; IPMM, índice padronizado pela média modificado; IPM, índice padronizado pela média; IZ, índice Z; e, SGP, seleção de ganhos positivos. PMV, produtividade de matéria verde; BRIX, sólidos solúveis totais; EXT, extração de caldo; TBH, massa de sólidos solúveis totais; POL, teor de sacarose no caldo. 
Tabela 5. Correlação de Spearman entre os índices (acima da diagonal) e percentagem de coincidência na seleção (abaixo da diagonal).

\begin{tabular}{lcccc}
\hline Índice & IR & IPMM & IPM & IZ \\
\hline IR & - & $-0,7145^{* *}$ & $-0,7145^{* *}$ & $-0,7061^{* *}$ \\
IPMM & 62,5 & - & $0,9900^{* *}$ & $0,9894^{* *}$ \\
IPM & 62,5 & 100,0 & - & $0,9883^{* *}$ \\
IZ & 62,5 & 90,0 & 90,0 & - \\
SGP & 52,5 & 72,5 & 72,5 & 80,0 \\
\hline
\end{tabular}

IR, índice de ranks; IPMM, índice padronizado pela média modificado; IPM, índice padronizado pela média; IZ, índice Z; e SGC, seleção de ganhos positivos. ${ }^{*}$ Significativo a $1 \%$ de probabilidade pelo teste $\mathrm{t}$.

apresentou coincidência de 90\% com os índices IPMM e IPM. O índice IR teve o valor de coincidência de $62,5 \%$ com todos os demais índices. Com base na seleção de ganhos positivos (SGP), houve coincidência de $52,5 \%$ com o IR, 72,5\% com os índices IPM e IPMM e $80 \%$ com o índice Z. Pedrozo et al. (2009) relataram que quanto maior o coeficiente de coincidência entre dois índices de seleção, maior será a concordância dos resultados de seleção entre eles.

No presente estudo, pôde-se observar que o uso de índices de seleção em sorgo-sacarino permite selecionar genótipos superiores a cada ciclo de seleção, com base em caracteres múltiplos.

\section{Conclusões}

1. Ganhos genéticos significativos de caracteres agronômicos, em progênies de sorgo-sacarino, podem ser observados por meio de seleção simultânea.

2. O índice padronizado pela média modificado (IPMM), o índice padronizado pela média (IPM) e o índice da soma dos valores padronizados (IZ) apresentam ganhos genéticos similares para todos os caracteres, quando se considera o ganho genético com base em genótipos superiores sobre a população original.

3. O índice de ranks (IR) apresenta os menores ganhos genéticos para todos os caracteres.

\section{Referências}

ANANDAN, S.; ZOLTAN, H.; KHAN, A.A.; RAVI, D.; BLÜMMEL, M. Feeding value of sweet sorghum bagasse and leaf residues after juice extraction for bio-ethanol production fed to sheep as complete rations in diverse physical forms. Animal Feed Science and Technology, v.175, p.131-136, 2012. DOI: 10.1016/j. anifeedsci.2012.05.005.
CARGNELUTTI FILHO, A.; STORCK, L. Medidas do grau de precisão experimental em ensaios de competição de cultivares de milho. Pesquisa Agropecuária Brasileira, v.44, p.111-117, 2009. DOI: $10.1590 / \mathrm{s} 0100-204 \times 2009000200001$

CONSECANA. Conselho de Produtores de Cana-de-Açúcar, Açúcar e Etanol do Estado de São Paulo. Manual de instruções. 5.ed. Piracicaba, 2006. 54p.

CRUZ, C.D.; CARNEIRO, P.C.S. Modelos biométricos aplicados ao melhoramento genético. Viçosa: Ed. da UFV, 2003. v.2, 585p.

CRUZ, C.D.; REGAZZI, A.J.; CARNEIRO, P.C.S. Modelos biométricos aplicados ao melhoramento genético. 4.ed. Viçosa: Ed. da UFV, 2012. v.1, 514p.

DURÃES, F.O.M. Sorgo sacarino: tecnologia agronômica e industrial para alimentos e energia. Agroenergia em Revista, ano.2, p.2-3, 2011.

FREITAS, I.L. de J.; AMARAL JÚNIOR, A.T. do M.; VIANA, A.P.; PENA, G.F.; CABRAL, P. da S.; VITTORAZZI, C.; SILVA, T.R. da C. Ganho genético avaliado com índices de seleção e com REML/Blup em milho-pipoca. Pesquisa Agropecuária Brasileira, v.48, p.1464-1471, 2013. DOI: 10.1590/S0100204X2013001100007.

GARCIA, A.A.F.; SOUZA JÚNIOR, C.L. de. Comparação de índices de seleção não paramétricos para a seleção de cultivares. Bragantia, v.58, p.253-267, 1999. DOI: 10.1590/S000687051999000200005.

GUIGOU, M.; LAREO, C.; PÉREZ, L.V.; LLUBERAS, M.E.; VÁZQUEZ, D.; FERRARI, M.D. Bioethanol production from sweet sorghum: evaluation of post-harvest treatments on sugar extraction and fermentation. Biomass and Bioenergy, v.35, p.3058-3062, 2011. DOI: 10.1016/j.biombioe.2011.04.028.

KRAUSE, W.; SOUZA, R.S. de; NEVES, L.G.; CARVALHO, M.L. da S.; VIANA, A.P.; FALEIRO, F.G. Ganho de seleção no melhoramento genético intrapopulacional do maracujazeiroamarelo. Pesquisa Agropecuária Brasileira, v.47, p.51-57, 2012. DOI: 10.1590/S0100-204X2012000100008.

LOMBARDI, G.M.R.; NUNES, J.A.R.; PARRELLA, R.A.C.; TEIXEIRA, D.H.L.; BRUZI, A.T.; DURÃES, N.N.L.; FAGUNDES, T.G. Path analysis of agro-industrial traits in sweet sorghum. Genetics and Molecular Research, v.14, p.1639216402, 2015. DOI: 10.4238/2015.December.9.8.

MENDES, F.F.; RAMALHO, M.A.P.; ABREU, A. de F.B. Índice de seleção para escolha de populações segregantes de feijoeirocomum. Pesquisa Agropecuária Brasileira, v.44, p.1312-1318, 2009. DOI: $10.1590 / \mathrm{S} 0100-204 X 2009001000015$.

MULAMBA, N.N.; MOCK, J.J. Improvement of yield potential of the Eto Blanco maize (Zea mays L.) population by breeding for plant traits. Egyptian Journal of Genetics and Cytology, v. 7, p.40-51, 1978.

PEDROZO, C.A.; BARBOSA, M.H.P.; RESENDE, M.D.V.; PETERNELLI, L.A.; COSTA, P.M. de A.; SILVA, F.L. da. Eficiência da seleção em fases iniciais do melhoramento da canade-açúcar. Revista Ceres, v.55, p.1-8, 2008.

PEDROZO, C.A.; BENITES, F.R.G.; BARBOSA, M.H.P.; RESENDE, M.D.V. de; SILVA, F.L. da. Eficiência de índices de 
seleção utilizando a metodologia REML/BLUP no melhoramento da cana-de-açúcar. Scientia Agraria, v.10, p.31-36, 2009. DOI: 10.5380/rsa.v10i1.11711.

PEREIRA, T.B.; MENDES, A.N.G.; BOTELHO, C.E.; REZENDE, J.C. da; VILELA, D.J.M.; DE RESENDE, M.D.V. de. Seleção de progênies $\mathrm{F}_{4}$ de cafeeiros obtidas de cultivares do grupo Icatu. Coffee Science, v.8, p.337-346, 2013.

RAMALHO, M.A.P.; ABREU, A. de F.B.; SANTOS, J.B. dos; NUNES, J.A.R. Aplicações da genética quantitativa no melhoramento de plantas autógamas. Lavras: Ed. da UFLA, 2012. 522p.

REIS, C.A.F.; GONÇALVES, F.M.A.; RAMALHO, M.A.P.; ROSADO, A.M. Seleção de progênies de eucalipto pelo índice Z por MQM e Blup. Pesquisa Agropecuária Brasileira, v. 46, p.517-523, 2011. DOI: 10.1590/S0100-204X2011000500009.

RESENDE, M.D.V. de. Software Selegen - REML/BLUP: sistema estatístico e seleção genética computadorizada via modelos lineares mistos. Colombo: Embrapa Florestas, 2007. 350p.

RESENDE, M.D.V.; DUARTE, J.B. Precisão e controle de qualidade em experimentos de avaliação de cultivares. Pesquisa Agropecuária Tropical, v.37, p.182-194, 2007.
RODRIGUES, F.; VON PINHO, R.G.; ALBUQUERQUE, C.J.B.; VON PINHO, E.V.R. Índice de seleção e estimativa de parâmetros genéticos e fenotípicos para características relacionadas com a produção de milho-verde. Ciência e Agrotecnologia, v.35, p.278-286, 2011. DOI: 10.1590/S141370542011000200007.

SILVA, G.A. da; PEREIRA, A. da S.; SOUZA, V.Q. de; CARVALHO, F.I.F. de; FRITSCHE NETO, R. Correlações entre caracteres de aparência e rendimento e análise de trilha para aparência de batata. Bragantia, v.66, p.381-388, 2007. DOI: 10.1590/S0006-87052007000300003.

TEIXEIRA, D.H.L.; OLIVEIRA, M. do S.P. de; GONÇALVES, F.M.A.; NUNES, J.A.R. Índices de seleção no aprimoramento simultâneo dos componentes da produção de frutos em açaizeiro. Pesquisa Agropecuária Brasileira, v.47, p.237-243, 2012. DOI: 10.1590/S0100-204X2012000200012.

VENCOVSKY, R. Herança quantitativa. In: PATERNIANI, E.; VIEGAS, G.P. (Ed.). Melhoramento e produção do milho. 2. ed. Campinas: Fundação Cargill, 1987. p.137-214.

WILLIAMS, J.S. The evaluation of a selection index. Biometrics, v.18, p.375-393, 1962. DOI: 10.2307/2527479.

Recebido em 16 de dezembro de 2015 e aprovado em 28 de junho de 2016 